\title{
Testing of local gamma-ray scatter fractions determined by spectral fitting
}

\author{
K F Koral†, X Wang†, K R Zasadny†, N H Clinthornet, W L Rogers $\dagger$, \\ C E Floyd Jrł and R J Jaszczak \\ $\dagger$ Division of Nuclear Medicine, University of Michigan, Ann Arbor, MI 48109, USA \\ $\ddagger$ Department of Radiology, Duke University Medical Center, Durham, NC 27710, \\ USA
}

Received 10 November 1989 , in final form 24 September 1990

\begin{abstract}
The spectral-fitting method of correction for gamma-ray Compton scattering within objects separates the unscattered and scattered components of locally measured energy spectra. Here, we employ a third-order polynomial for the scattering and an approximately constant fitting window. A scatter fraction, defined as total scattered over total unscattered counts within a $20 \%$ window, is calculated for each point in our Anger camera iniages. These scatter fractions are tested against those from Monte-Carlo simulation for ${ }^{99 \mathrm{~m}} \mathrm{Tc}$ and against results from semiconductor detector measurement.s for ${ }^{131} \mathrm{I}$. A radioactive sphere at. several locations within a non-radioactive cylinder and the inverse are imaged for the testing. For one case, reproducibility of the spectral-fitting scalter fraction as a function of the number of unscattered counts within the $20 \%$ acceptance window was also determined. With ${ }^{99} \mathrm{~m} \mathrm{Tc}$, for all cases, the agreement between spectral fitting and the standard estimation method is within $16 \%$. With ${ }^{131} \mathbf{l}$, for the 'hot' sphere at two locations, the agreement is within $21 \%$. For the 'hot' sphere at the third location (off the cylinder axis towards the camera), the dependence of scatter fraction on transverse distance is good although the absolute values are too large. Scatter fraction reproducibility is within $10 \%$ for 1000 or more counts. Therefore, further testing of spectral fitting and initial application to realistic clinical images seem to be in order.
\end{abstract}

\section{Introduction}

Compton scattering is one of the three major problems in single photon emission computed tomography (SPECT) that must be addressed to achieve quantitative accuracy (attenuation and finite-resolution effects are the others). Among techniques for simply reducing detection of scattered gamma rays are energy windows that are offset (Koral et al 1986) or reduced in width (Leichner et al 1987). Accuracy is improved as the scatter component is reduced, but statistical uncertainty is increased since the number of unscattered photons is also reduced. The energy-weighted acquisition method (Hamill et al 1989) is an on-line correction technique which is currently in use with Anger cameras (Halama et al 1988), but its quantitative accuracy has not as yet been established.

Among other techniques that correct for Compton scattering are those of Egbert and May (1980), Beck et al (1982), Bergström et al (1983), Axelsson et al (1984), Jaszczak et al (1984), Todd-Pokropek ct al (1984), Msaki et al (1985, 1987), Floyd 
et al (1985a, b), Koral et al (1988), Singh and Horne (1988), Yanch et al (1988) and Gagnon et al (1989). The basic hypothesis for almost all the correction methods is the additive model, i.e. the detected counts in a certain energy window are the sum of unscattered counts (also called direct counts) and scattered counts. Therefore, the major task for scatter correction is to estimate the scattered counts in the window, and once that is done, a simple subtraction provides the corrected results.

Among the proposed methods, those of Koral et al (1988) and Gagnon et al (1989) estimate the scattered comts by analysing the complete energy spectrum at individual spatial locations in the image. They have both been shown to be feasible. The purpose of this work is to assess further the spectral-fitting scatter correction technique (Koral et al 1988) in the case of fairly good counting statistics. It is compared with test methods for several different phantom geometries. Also checked is the reproducibility of the technique.

\section{Methods}

To evaluate and compare methods for scatter correction, we use the accuracy of the scatter fraction, SF. For a certain energy window, SF is defined as

$$
\mathrm{SF}=S / U
$$

where $S$ and $U$ are the total number of scattered and unscattered counts within the window, respectively. Note that, defined in this usual way, SF can be greater than 1 . Such a value does not imply that gamma rays have been created.

\subsection{Analysis of energy spectra (fitting method)}

To permit analysis of local Anger camera spectra (those at specified locations in the image of the object), an 'energy spectrum image' is taken. This image is a determination of the energy spectrum over a given energy range at every $x, y$ location in the image. Then, for each individual energy spectrum, the unscattered component is represented by a scatter-free spectrum (separately measured, see later discussion) times a constant, and the Compton-scattered component is represented by a low-order polynomial (always a cubic in this study). The sum of the two components is then fitted to the measured spectrum to get both the constant and the polynomial coefficients, and thus the total counts from each component in the given energy window. To reduce the amount of computation and to improve statistics, an individual spatial location is usually a small region of interest instead of a single pixel. Hereafter, this method will be referred to as the 'fitting method' for obvious reasons.

For this study, the 'energy spectrum image' was obtained in the following way: a very narrow (2 keV for ${ }^{99 \mathrm{~m}} \mathrm{Tc}, 3 \mathrm{keV}$ for ${ }^{131} \mathrm{I}$ ) window was set at the lowest energy of interest, 108 to $110 \mathrm{keV}$ for ${ }^{99 \mathrm{~m}} \mathrm{Tc}$ and 300 to $303 \mathrm{keV}$ for ${ }^{131} \mathrm{I}$. An image of the object was acquired for a fixed time and stored. The window was then advanced to the next level (110 to $112 \mathrm{keV}$ or 304 to $307 \mathrm{keV}$ ) and an acquisition obtained. This procedure was repeated 32 times in all. The number of counts at any given position in the image was plotted against energy to obtain the desired spectrum.

From here on, each narrow window is referred to as an energy channel and numbered from 1 to 32 . One set of two channel numbers, $m_{1}$ and $m_{2}$, called the fitting 
window, is used for the energy range over which the fitting is to be performed. This fitting window was kept fixed for each of the three experiments and was always symmetrically wider than the summation window (described below). The average difference, $n_{1}-m_{1}$ (with $n_{1}$ defined below), for the three experiments was $4.7 \%$ of $E$, the energy of the direct gamma ray (range 4.1 to $5.7 \%$ ). Although the scatter fractions and the values for unscattered count rate may vary with the fitting window (and with the polynomial order), we have kept these approximately fixed to evaluate the technique in its simplest form.

The scatter fraction, SF, is calculated by

$$
\mathrm{SF}=\left(\sum_{i=n_{1}}^{n_{2}} S_{i}\right)\left(\sum_{i=n_{1}}^{n_{2}} U_{i}\right)^{-1}
$$

where $S_{i}$ is the Compton-scattered counts and $U_{i}$ is the unscattered counts in channel $i$ and the summation window is determined by channel numbers $n_{1}$ and $n_{2}$. These values are chosen to produce a $20 \%$ window: from $0.90 \times E$ to $1.10 \times E$. For ${ }^{99 m} \mathrm{Tc}$, the mechanism for setting $n_{1}$ and $n_{2}$ involved measuring the dependence of channel number on energy by a calibration employing the centroid of the energy peaks from ${ }^{133} \mathrm{Xe}(81.0 \mathrm{keV})$ and ${ }^{111} \mathrm{In}(171.2 \mathrm{keV})$. For ${ }^{131} \mathrm{I}$, we used the peak channel as $364 \mathrm{keV}$ and assumed the nominal $\mathrm{keV} / \mathrm{channel}$ was accurate.

Readers should see Koral et al (1988) for details about the mathematics of the least-squares fitting itself.

\subsection{Monte-Carlo Simulation}

Floyd et al studied Compton scattering in SPECT using Monte-Carlosimulation (Floyd et al 1984). In this paper, the same computer simulation program is used. The exact phantom geometry and activity distribution is modelled by this program. Energy spectra as a function of position in the camera image are built up gradually by the code. Tracking of an individual photon is terminated when its energy falls below the low-energy threshold of the fitting window, taking into account the camera energy resolution. Scatter fractions are calculated from eqaution (2). Judging by the average agreement between results for symmetric positions, the uncertainty is $\pm 0.1 \%$ for the 'hot' sphere (section 3.3 ) and $\pm 3.9 \%$ for the fewer-photon-histories 'cold' sphere (section 3.4).

Higher-energy photon simulation is not possible with the present program as it does not model collimator septal penetration. Therefore, an alternative means is necessary for verification of the fitting method for the photons of ${ }^{131} \mathrm{I}$.

\subsection{Semiconductor-detector measurements}

Scatter fraction measurements have been made using a high-purity germanium (HPGe) detector for both ${ }^{99} \mathrm{~m} \mathrm{Tc}$ and ${ }^{131} \mathrm{I}$ (Zasadny et al 1990). The HPGe method has been validated for a hot sphere filled with ${ }^{99 \mathrm{~m}} \mathrm{Tc}$ solution in a water-filled cylinder by MonteCarlo simulation in that reference. It is, therefore, assumed in this paper that HPGe scatter-fraction measurements for a hot sphere filled with ${ }^{131}$ I solution are sufficiently accurate to provide a test of the spectral-fitting method.

A brief summary of the HPGe detector method is as follows. The good energy resolution of the detector almost enables one to seperate directly the scattered photon events from the unscattered events by finding the energy that divides the two. To 
account for slight spillover across this energy due to the detector's finite resolution, spectral fitting is employed, utilizing a scatter-free spectrum (see section 2.4). The magnitude of the change in the scatter fraction from this spillover is less than $6.4 \%$ of the value. The result is a separation of the raw data spectrum into scattered and unscattered spectra. A measured energy calibration then sets the upper and lower energy corresponding to a $20 \%$ window. Finally, we compute Anger camera energy spectra by convolving with the appropriate Anger camera energy response function ai each energy. Then, eqaution (2) is applied to yield the scatter fraction expected with an Anger camera. Again, by comparing results for symmetric positions, the uncertainty for this standard is $\pm 7.8 \%$ (section 3.5 ).

\subsection{Scatter-free spectrum}

Originally, in Koral et al 1988, the scatter-free spectrum used for fitting was that for a small syringe, containing radioactive isotope, placed in the air in front of the camera. The spectrum still had a low-energy tail. Recently, Anger camera experiments have shown that a scatter-free spectrum made with a disk-like filter paper, with a diameter of about $2 \mathrm{~cm}$, saturated with a solution of radioactive isotope, has a $50 \%$ lower tail than the syringe spectrum due to less scattering. Since the model depends on the scatter-free spectrum to be what its name implies, measurements of these spectra are made with such a filter paper source throughout this study.

\subsection{Correction for camera dead time}

To reduce data acquisition time with the Anger camera and to prevent drift of the camera electronics, while still maintaining good statistics, the sources used were quite strong, usually 10 to $20 \mathrm{mCi}$. The (observed) peak count rate in a $2 \mathrm{keV}$ window reached 20000 counts per second. The dead time of the camera under such a count rate might significantly affect the shape of the energy spectra of the measured object, and thus change the calculated scatter fraction. Therefore, it was necessary to be able to correct the measured energy spectra for dead time.

The method used to check dead time is that of Freedman et al (1972). A very weak source, called the monitor source, is put on the camera face near the edge, and covered with lead to prevent it from being interfered with by the experimental object. Then, the experiments are carried out as usual except that after acquisition an extra energy-spectrum image of the monitor source alone is taken. We assume that the dead time for the monitor-alone energy spectrum is negligible since the source is very weak. For each energy channel, we find the counts in a small region of interest surrounding the monitor source for both the image with only the monitor source and also that with the monitor source and object. These counts are corrected for isotope decay during the time between images. The ratio then gives the factor for dead time correction.

\subsection{Differencing measurement}

Recently, the results for a ${ }^{99 \mathrm{~m} r} \mathrm{Tc}$ point source in air were subtracted from those for the source in water to determine the scatter fraction for the entire image (Knesaurek et al 1989). The authors derived the following formula:

$$
\mathrm{SF}=0.065 \mathrm{~cm}^{-1} \times d
$$

for a $20 \%$ energy window, where $d$ is the depth of the point source in water. As an additional part of this paper, the scatter fraction of the whole image for a radioactive sphere is calculated and compared with the formula. 


\subsection{Experiments}

The phantom used for all measurements is a water-filled cylinder containing a $6 \mathrm{~cm}$ diameter sphere. Figure 1 shows the geometry. The cylinder has a diameter of $19.7 \mathrm{~cm}$ and a height of $25.4 \mathrm{~cm}$. The sphere can take one of three positions inside the cylinder: one is on the axis of the cylinder (the origin of the $x-y-z$ coordinate system shown in figure 1), the other two are $5.7 \mathrm{~cm}$ from the axis toward or away from the camera face along the $z$ axis. These three positions will hereafter be referred to as 'on-axis', 'near' and 'far', respectively. The phantom is either a 'hot sphere', i.e. the sphere containing the solution of radioactive isotope in a cylinder filled with non-radioactive water, or a 'cold sphere' which is the inverse.

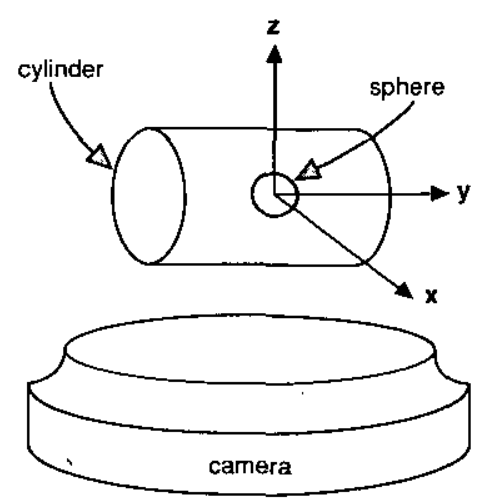

Figure 1. The experimental geometry. The cylinder was at a fixed position near the face of the collimator. The sphere took three positions with its centre on the axis of symmetry of the cylinder and with it at an equal distance nearer to or farther from the camera. The origin of the coordinate system moves with the sphere.

The Anger camera used in the experiments is a GE 400AT. The energy resolution (FWHM) of the camera is about $10.8 \%$ for ${ }^{99 \mathrm{~m}} \mathrm{Tc}$ with a low-energy, general purpose collimator, and about $9.5 \%$ for ${ }^{131}$ I with a high-energy collimator. The pixel size for both is about $3 \mathrm{~mm}$ ( 128 by 128 acquisition).

Three experiments are conducted. The first is for a ${ }^{99 \mathrm{~m}} \mathrm{Tc}$ hot sphere at all three positions. The SF results along the $x$ axis are compared with Monte-Carlo simulation. The second is for a Tc cold sphere. Only the near position is tested, but the comparison with Monte-Carlo simulation is made along both the $x$ and $y$ axes. Lastly, a ${ }^{131}$ I hot sphere is measured at the three different positions with both the Anger camera and the HPGe semiconductor detector.

For the HPGe measurement, the source is collimated by a high-energy Anger camera collimator and the active area of the detector is defined by a $0.6 \mathrm{~cm}$ diameter hole in a lead shielding mask. The thickness of the mask is $5.7 \mathrm{~cm}$. Scans at various locations across the diameter of the phantom are made by translating the source. A measurement of a scatter-free source and an energy calibration are also taken. Finally, the detector cfficicncy as a function of photon energy is measured by counting an accurately calibrated multiple-energy photon source obtained from the National Bureau of Standards. All spectra are initially corrected to account for the varying efficiency.

Also tested is the reproducibility with the ${ }^{131}$ I hot sphere in the on-axis position. To do this, two sets of Anger camera data are taken and the total unscattered counts, 
$U_{1}$ and $U_{2}$, are estimated from fitting. The second result is corrected for the decay of the source between the two measurements. Then, the reproducibility of the result is found by calculating the percentage difference, defined as

$$
\text { percentage difference }=\frac{\left|U_{1}-U_{2}\right|}{\left[U_{1}, U_{2}\right]} \times 100 \%
$$

where [] is the averaging operator.

\section{Results}

\subsection{The effect of dead time correction}

The dead time correction factor with the hot sphere was largest when it was in the near position. Figure 2 shows the correction factor plotted against energy for the near ${ }^{131} \mathrm{I}$ measurement. As can be seen in the figure, the factors are as high as 1.5 to 1.6 in the peak channels. Table 1 shows the effect of such a dead time correction on the calculated scatter fractions with a ${ }^{99 \mathrm{~m}} \mathrm{Tc}$ hot sphere in the near position. There the effect of making the correction is to decrease SF by an average $25 \%$. Since dead time correction is significant in at least some cases, it has been applied to all the data in this study.

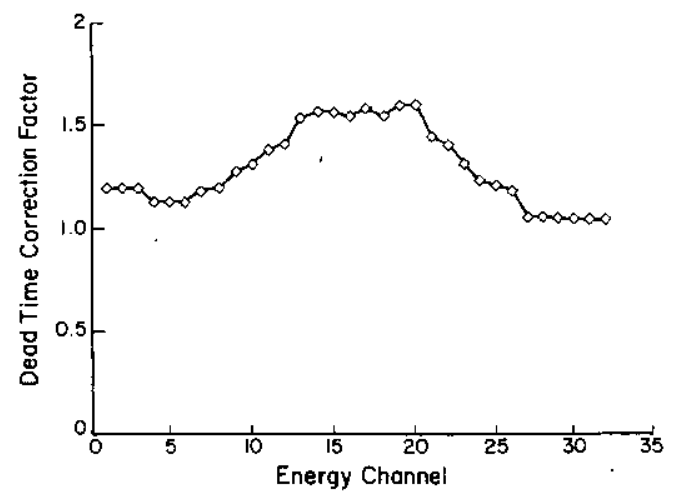

Figure 2. Deadtime correction factor plotted against energy for the near ${ }^{131}$ I measurement. The relation between channel and energy is given by Energy $(\mathrm{keV})=4 \times$ Channel +304 . The largest corrections correspond to the photopeak as can be seen by comparing with figure 3 .

Table 1. Effect of dead time correction on scatter fraction for ${ }^{99 \mathrm{~m}} \mathrm{Tc}$ hot sphere in near position where correction is large.

\begin{tabular}{lcccccc}
\hline & \multicolumn{7}{c}{$x$ location (mm) } \\
\cline { 2 - 7 } & -30.75 & -18.45 & -6.15 & 6.15 & 18.45 & 30.75 \\
\hline Without dead time correction & 0.827 & 0.295 & 0.280 & 0.246 & 0.245 & 0.628 \\
With dead time correction & 0.634 & 0.221 & 0.211 & 0.181 & 0.180 & 0.476 \\
Percentage change & 23.3 & 24.7 & 24.6 & 26.4 & 26.5 & 24.2 \\
\hline
\end{tabular}




\subsection{Fitting spectra}

Three fitted spectra for the ${ }^{131} 1$ hot-sphere are shown in figure 3 . The abscissa of the plot is bounded by the fitting window $\left(m_{1}\right.$ and $m_{2}$ ). The summation window $\left(n_{1}\right.$ and $n_{2}$ ) is also shown. Going from (a) to (c), one moves along $x$, that is, from considering the area opposite the edge of the hot sphere (larger scatter fraction) to considering that opposite the centre (smaller scatter fraction). These results indicate that good fits can be obtained over a range of scatter fractions.

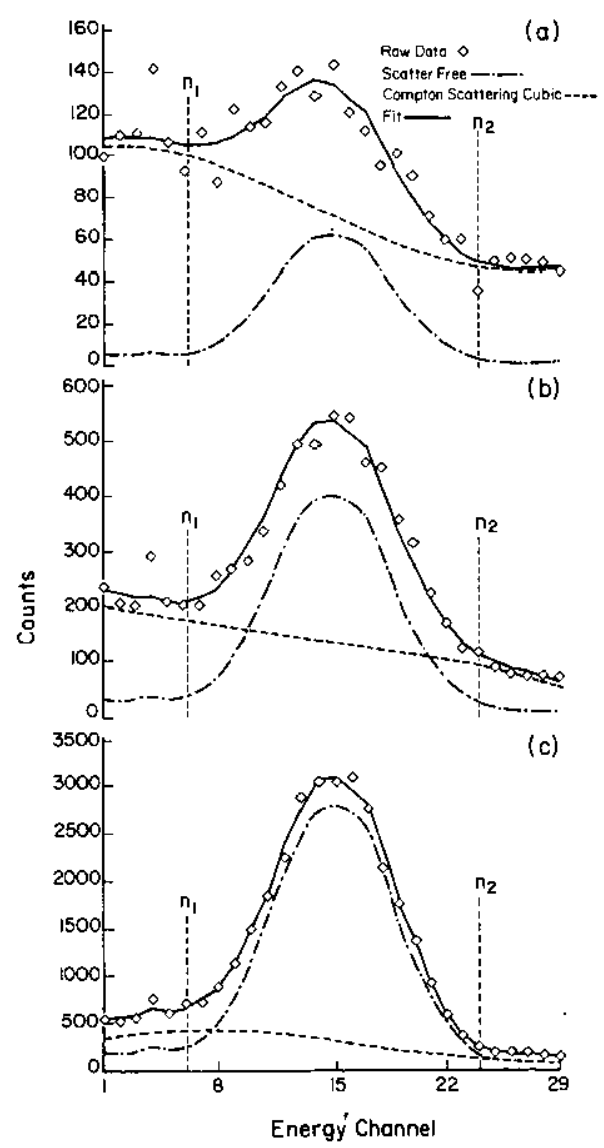

Figure 3. Examples of ${ }^{131}$ I spectral fitting where the scatter fraction is found to be (a) large, (b) medium and (c) small. The scatter-free spectrum as shown has been multiplied by a constant determined by the fit. The $n_{1}$ and $n_{2}$ define the $20 \%$ summation window for the scatter fractions reported in the paper.

\section{3. ${ }^{99 m}$ Tc hot sphere}

3.3.1. Comparison with monte-carlo simulation. Figures 4(a)-(c) compare the SF from fitting for different points along the $x$ axis with that from Monte-Carlo simulation for the far, on-axis and near positions of the sphere, respectively. The size of each spatial location is 4 by 4 pixels, or about $12.3 \mathrm{~mm}$ by $12.3 \mathrm{~mm}$. The fitting window is \pm 3 
channels wider than the $20 \%$ window. The figures show a fairly flat distribution until one gets almost outside the region of activity where scatter still exists and the SF therefore goes up.

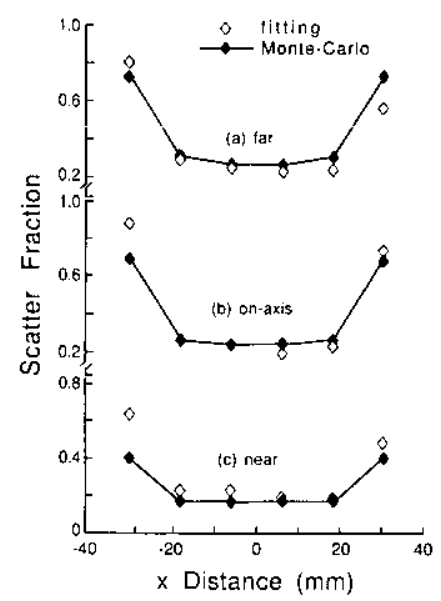

Figure 4. Comparison of Monte-Carlo and spectral-fitting scatter fractions for points in the image that project onto the $x$ axis through the object. This axis passed through the centre of the sphere as in figure 1. The sphere contains ${ }^{99} \mathrm{~m} \mathrm{Tc}$ in solution and for the three cases its centre was positioned (b) on the axis of symmetry of the cylinder (as shown in figure 1), (a) $5.5 \mathrm{~cm}$ farther from the camera and (c) $5.5 \mathrm{~cm}$ nearer to the camera. The cylinder contains non-radioactive water.

It is seen that the agreement is generally good (some fitting points in the graph are actually obscured by the Monte-Carlo symbols). The average over all points of the absolute value of the difference between spectral fitting and Monte-Carlo divided by Monte-Carlo is $16.2 \%$. This difference is slightly larger than the average uncertainty in the fitting results, which is computed as $\pm 10.1 \%$ by comparing symmetric positions.

3.3.2. Spectrum of entire image. The first two rows of table 2 compare the scatter fraction from the fitting method with Knesaurek's formula. The results show very good agreement. This interesting correspondence is not meant to say that a point source and a $6 \mathrm{~cm}$ diameter sphere necessarily have the same total-image scatter fraction, but only that an independent experiment finds scatter fractions very similar to ours for at least a similar geometry.

Table 2. Experimental scatter fractions for spectrum of entire image with hot object within a cold cylinder.

\begin{tabular}{lllll}
\hline & & \multicolumn{3}{c}{$\begin{array}{c}\text { Position of point source } \\
\text { or sphere centre }\end{array}$} \\
\cline { 3 - 5 } Method & Isotope & near $5.1 \mathrm{~cm} \dagger$ & on-axis $10.8 \mathrm{~cm} \dagger$ & far $16.5 \mathrm{~cm} \dagger$ \\
\hline Knesaurek's formula & $99 \mathrm{~m} \mathrm{Tc}$ & 0.332 & 0.702 & 1.07 \\
Fitting & $99 \mathrm{~m} \mathrm{Tc}$ & 0.311 & 0.748 & 1.02 \\
Fitting & $131 \mathrm{I}$ & 0.554 & 0.696 & 0.816 \\
\hline
\end{tabular}

† Depth from edge of cylinder near camera. 
The true scatter fraction really varies spatially according to our findings. One can check the importance of this variation for scatter correction. Using the spectral-fitting scatter fractions, one can calculate the error in direct counts one makes for different situations if one uses only the whole-image scatter fraction. A region of interest is chosen for the whole sphere from considering the pixel size. The results are listed in table 3. The error increases rapidly as the sphere is located deeper in the cylinder (far from the camera). There, the SF from the whole image is too large and not representative of that for the sphere.

Table 3. Percent errort in hot-sphere unscattered count when scatter fraction of the whole image is used for Compton correction.

\begin{tabular}{lccc}
\hline & \multicolumn{3}{c}{ Sphere position } \\
\cline { 2 - 4 } & near & on-axis & far \\
\hline At sphere centre & -7.82 & -28.6 & -37.8 \\
For whole sphere & -4.93 & -24.7 & -34.3 \\
\hline
\end{tabular}

$\dagger$ Error is calculated direct count from whole-image sF minus calculated direct count. from locally measured $S F s$, divided by the latter.

\section{4. ${ }^{99 m}$ Tc cold sphere}

Figures 5(a) and (b) compare the SF from fitting and Monte-Carlo in the $x$ and $y$ directions with the cold sphere near. The fitting window is \pm 4 channels wider than the $20 \%$ window. The transverse distance covered is greater than before. The SF values show a broad maximum in the region over the sphere and a slight decrease toward the edge of the cylinder.

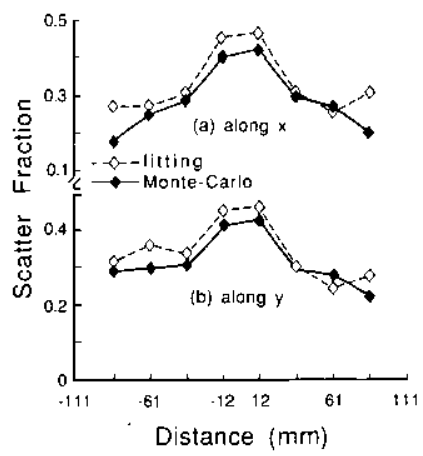

Figure 5. Comparison of Monte-Carlo and spectral-fitting scatter fractions for points in the image that project onto (a) the $x$ axis and (b) the $y$ axis through the object. Here, the cylinder contains ${ }^{99 \mathrm{~m}} \mathrm{Tc}$ in solution and the sphere non-radioactive water. The sphere was in the near position.

Except for two points, the fitting results are slightly larger than those from MonteCarlo. Overall, the average difference, between fitting and standard, $15.3 \%$, is larger than the average uncertainty in the fitting results, computed as $\pm 5.6 \%$. However, here the standard also has a non-negligible average uncertainty of $\pm 3.9 \%$ so the agreement is only slightly worse than with the hot sphere. 


\section{5. ${ }^{131}$ I hot sphere}

The scatter fractions from fitting the spectrum for the entire image are shown in the last line of table 2 . They change less rapidly with depth than they did with ${ }^{99 \mathrm{~m}} \mathrm{Tc}$ as one might expect for the more penetrating gamma ray from ${ }^{131} \mathrm{I}$.

3.5.1. Comparison with semiconductor-detector results. Comparison of the scatter fractions from fitting with those from the semiconductor detector are shown in figure 6 . The data were grouped so that the pixel size is $2 \times 2$ or about $6.1 \mathrm{~mm}$ on a side. The fitting window is \pm 5 channels wider than the $20 \%$ window. Overall, the average difference between fitting and semiconductor detector in $6(\mathrm{a})$ and (b), 21.3\%, is larger than the average uncertainty in the fitting results, estimated as $\pm 4.6 \%$. However, here the error from the standard is estimated to be as high as $\pm 7.8 \%$ so agreement is similar to that with ${ }^{99 \mathrm{~m}} \mathrm{Tc}$.

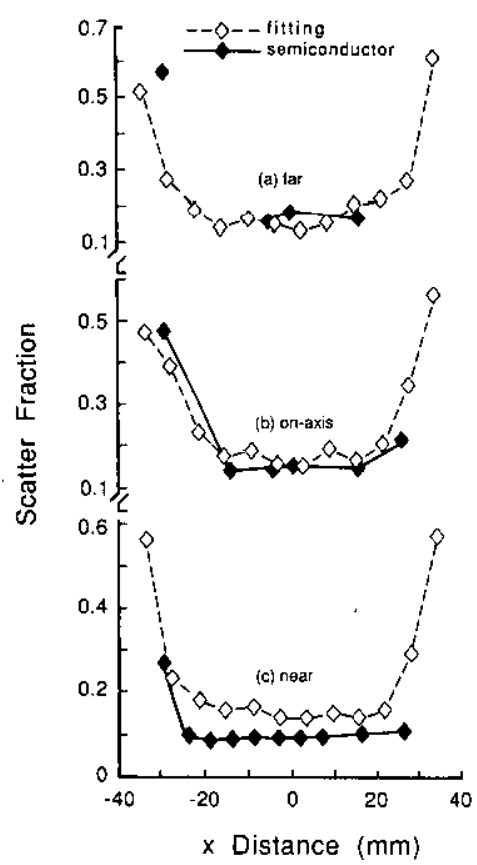

Figure 6. Comparison of semiconductor detector and spectral-fitting scatter fractions for points in the image that project onto the $x$ axis through the object. The sphere contains ${ }^{131} \mathrm{I}$ in solution and its centre was positioned (a) far, (b) on-axis and (c) near as in figure 4. The cylinder again contains non-radioactive water. In (a), no semiconductor detector results were obtained between -47 and -30 mm nor beyond $+15 \mathrm{~mm}$.

In figure $6(\mathrm{c})$, the fitting method consistently gives larger values than does the semiconductor detector with an average difference of $74.1 \%$. Although a systematic error cannot be ruled out, it appears that here the given spectral-fitting algorithm has failed to produce accurate results.

3.5.2. Reproducibility test. The percentage difference was calculated by eqaution (4) for all spatial locations. Since the phantom is approximately spherically symmetric, the calculated differences were averaged according to their position relative to the 
centre of the sphere. They are plotted against the average unscattered counts in figure 7. Also shown in the figure is the average scatter fraction for each group. This value changes with the changes in distance from the centre of the sphere. The reproducibility is quite good when the average unscattered count is above 5000 (and the SF less than 0.5$\}$, presumably because then the statistical error for the number of counts in each energy channel is relatively small. The difference increases rapidly as the unscattered count decreases below 5000 (and the SF increases), being still at about $10 \%$ for only 1000 counts at a scatter fraction of 1.7 . Below that, it becomes unacceptable.

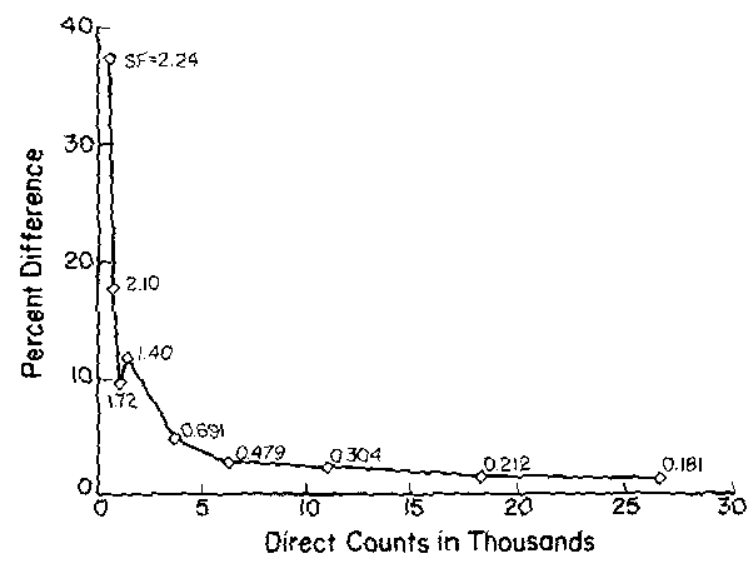

Figure 7. Reproducibility of fitting method for case (b) of figure 6. Several symmetrically located image points have their scatter fraction values, percentage differences and counts averaged to produce a value for the curve. The pertentage difference increases as the direct count decreases as one is examining points farther from the sphere centre with a smaller projection length through the active volume. The average scatter fraction is also increasing consistent with the pattern along a projected radius (figure 63. Although the scatter fraction is not fixed, it appears that one may surmise that decreased direct count (and thus increased statistically error) leads to increased percentage difference (that is, worse reproducibility) as one would expect.

\section{Discussion}

We were interested in applying spectral fitting when the energy spectra were not corrupted by large errors from counting statistics. For practical reasons, this interest required the use of strong sources. Such use necessitated channel-by-channel dead time correction. It is difficult to estimate the errors in the correction factors themselves, but by comparing results for symmetric positions we estimate the uncertainty in the final scatter fractions to be $10 \%$ or less. The alternative experimental approach would have been to wait for the development of a new, more efficient energy spectrum image acquisition system where dead time could be lower, but we chose to avoid delay.

In the one case where there is disagreement between the spectral-fitting result and that from the 'gold standard', it is possible that there was a systematic error in the standard or in the dead time correction. It is more likely, in our opinion, that a perturbation in the parameters of spectral fitting (order of polynomial or width of 
fitting window) is required. If so, an improved algorithm might require the parameters to vary with the imaging situation. Further investigation of these parameters is needed.

However, spectral fitting with a third-order polynomial and an approximately constant fitting window agrees with the 'gold standard' within an average difference of $17.6 \%$. This difference is not too much larger than the sum of the fitting and standard uncertainty in seven diverse cases out of eight. The standard is a completely different method in the case of Monte-Carlo simulation and involves a completely different detector in the case of the HPGe measurements. Therefore, we characterize this basic application of the spectral-fitting algorithm as producing generally accurate values although further testing is needed.

Note that the actual shape of the spectrum of unscattered gamma rays was not compared between methods. However, since spectral fitting is a technique for correction of effects from Compton scattering, the accuracy of the number of unscattered gamma rays is of primary importance even if there would be compensating errors in the spectrum. The accuracy of the scatter fraction is a sensitive test for the accuracy of this number so the comparisons presented above are important ones.

Since the output value when spectral fitting is used is the number of unscattered counts, we looked at variations in these counts to carry out the reproducibility test. The percentage difference for unscattered counts in the vicinity of the ${ }^{131}$ I sphere was $10 \%$ or less when 1000 counts or more were available. This test was not ideal because the scatter fraction was not held constant. If further testing gives similar results, it appears that, in most cases, fine pixels will have to be grouped and, in SPECT, angles combined, to accumulate the necessary counts in a spectrum. However, it is known that the Compton scattering image is inherently smooth in space and angle. Therefore, it does not need to be sampled very finely. One can smooth the results from the grouped points and interpolate between them to obtain the sampling density in space and angle of the original data. Scatter subtraction will then be carried out with these values.

In summary, the results in this paper are encouraging. The fitting method for correction of Compton scattering has been shown to be not only feasible but also generally accurate and reliable. The initial testing of this method on realistic clinical images to evaluate resultant SPECT quality and quantitative accuracy is thus suggested.

\section{Acknowledgments}

This work was supported by PHS Grants Nos R01 CA38790, R01 CA32846 and P0 CA42768 awarded by the National Cancer Institute, DHHS.

\section{Résumé}

Test des fractions des diffusé de rayonnemenets gamma locales déterminées par ajustement de spectres.

La méthode d'ajustement de spectres pour la correction du rayonnement diffusé Compton dans des objets sépare les composantes diffusées et non diffusées des spectes d'énergie mesurés localement. Dans ce travail, les auteurs utilisent un polynome de troisième ordre pour le diffusé et une fenêtre d'ajustement approximativement constante. Une fraction de diffusé définie, comme le rapport due diffusé total sur le nombre d'événements total non diffusés dans une fenêtre de $20 \%$, est calculée pour chaque point des images de la caméra Anger. Ces fractions de diffusé sont comparées à partir d'une simulation de Monte-Carlo pour du technecium $99 \mathrm{~m}$ et à partir des résultats de mesures par 
détecteur semi-conducteur pour l'iode 131. Une sphère radioactive située en plusieurs positions dans un cylindre non radioactif, et l'inverse, sont imagés pour le test. Pour un des cas, les auteurs ont également déterminé la productibilité de la fraction du diffusé obtenu par ajustemenet de spectre en fonction du nombrc d'évènements non diffusés dans la fenêtre de $20 \%$. Avec le technecium $99 \mathrm{~m}$, dans tous les cas, l'accord entre l'ajustement spectral et la méthode d'estimation standard est meilleure que $16 \%$. Avec l'iode 131, pour la sphère 'chaud' en deux positions, l'accord est meulleur que $21 \%$. Pour la sphère 'chaud' en troisième position (hors de l'axe du cylindre vers la gamma caméra), la dépendance de la fraction des diffusé vis-à-vis de la distance transverse est bonne bien que les valeurs abolues soient trop grandes. La reproductibilité de la fraction de diffusé est meuilleure que $10 \%$ pour 1000 évènements et plus. Ainsi, des tests complémentaires de l'ajustement spectral et l'application initiale à des images cliniques réalistes semblent être faisables.

\section{Zusammenfassung}

Überprüfung lokaler Gammastrahlen-Streuanteile mit Hilfe spektraler Anpassungsmethoden.

Die spektrale Anpassungsmethode zur Korrektur der Comptonstreuung innerhalb von Objekten trennt die gestreuten und die nicht gestreuten Komponenten lokal-gemessener Energjespektren. In der vorliegenden Arbeit wird ein Polynom dritter Ordnung für die Streuung und ein näherungsweise konstrantes Anpassungsfenster verwendet. Ein Streuanteil, definiert als Gesamt-Streuimpulse zu den gesamten nicht-gestreuten Impulsen innerhalb eines $20 \%$ Fensters, wird berechnet für jeden Punkt in den Anger-Kamera-Bildern. Diese Streufunktionen werden verglichen mit jenen aus ${ }^{99 \mathrm{~m}} \mathrm{Tc}$ Monte Carlo Simulationen und mit Ergebnissen von Halbleiter-Detektormessungen für ${ }^{131} \mathrm{I}$. Eine radioaktive Kugel wurde an verschiedenen Stellen innerhalb eines nicht-radioaktiven Zylinders zu Testzwedken abgebildet. In einem Fall wurde auch die Reproduzicrbarkeit des spektral angepassten Streuanteils als eine Funktion der Anzahl der nicht-gestreuten Impulse innerhalb des $20 \%$ Fensters bestimmt. Für $99 \mathrm{~m}$ Tc liegt in allen Fällen die Übereinstimmung zwischen spektraler Anpassung und der Standardbestimmungsmethode ionnerhalb $16 \%$. Bei ${ }^{131} \mathrm{I}$ liegt die Übereinstimmung für die radioaktive Kugel an zwei Stellen innerhalb 21\%. An der dritten Stelle (neben der Zylinderachse in Richtung Kamera) ist die Übereinstimmung zwischen spcktraler Anpassung und der Standardbestimmungsmethode gut, obwohl die absoluten Werte zu groß sind. Die Reproduzierbarkeit des Streuanteils liegt innerhalb 10\% für 1000 oder mehr Impulse. Deshalb sind wetere Tests der spektralen Anpassung, swoie die Anwendung auf realist sche klinische Bilder notwendig.

\section{References}

Axelsson B, Msaki P and Israelsson A 1984 Subtraction of Compton-scaltered photons in emission computed tomography J. Nucl. Med. 25 490-4

Beck J W, Jaszczak R I, Coleman R E, Starmer C F and Nolte L W 1982 SPECT including scatter and attenuation using sophisticated Monte-Carlo modeling methods IEEE Trans. Nucl. Sci. NS-29 506-11

Bergström M, Eriksson L, Bohm C, Blomqvist G and Litton J 1983 Corrections for scattered radiation in a ring detector positron camera by integral transformation of the projections $J$. Comput. Assist. Tomogr. $742-50$

Egbert S D and May R S 1980 An integral-transport method for Compton-scatter correction in emission computed tomography IEEE Trans. Nucl. Sci. NS-27 543-8

Floyd C E, Jaszczak RJ, Greer K L and Coleman R E 1985a Deconvolution of Compton scatter in SPECT J. Nucl. Med. 26 403-8

Floyd C E, Jaszczak R J, Harris C C, Greer K L and Coleman R E 1985b Monte-Carlo evaluation of Compton scatter subtraction in single photon emission computed tomography Med. Phys. 12 776-8

Frecdman G, Kinsela $\mathrm{T}$ and Dwyer A 1972 A correction method for high-count-rate quantitative radionuclide angiography. Radiology, 104 713-5

Gagnon D, Todd-Pokropek A, Arsenault A and Dupras G 1989 Introduction to holospectral imaging in nuclear medicine for scatter subtraction IEEE Trans. Med. Imag. 8·245-50

Hamill $\mathrm{J} J$ and Devito R P 1989 Scatter reduction with energy-weighted acquisition. IEEE Trans. Nucl. Sci. 26(2) 1334-9 
Halama J R, Henkin R E and Friend L E 1988 Gamma camera radionuclide images: Improved contrast with energy-weighted acquisition. Radiology, $169533-8$

Jaszczak R J, Greer K L, Floyd C C and Coleman R E 1984 Improved SPECT quantification using compensation for scattered photons J. Nucl. Med. 25 893-900

Knesaurek K, King M A and Glick S J 1989 Two methods of scatter fraction measurement in SPECT imaging J. Nucl. Med. suppl. 30876 (abstract)

Koral K F, Clinthorne N H and Rogers W L 1986 Improving emission computed tomography by Compton scatter rejection through offset windows Nucl. Instrum. Meth. Phys. Res. A $242610-$ 4

Koral K F, Wang X, Rogers W L, Clinthorne N H and Wang X 1988 spect Compton-scattering correction by analysis of energy spectra $J$. Nucl. Med. 29 195-202

Leichner P K, Yang N C, Frenkel T L, Loudenslager D M, Hawkins W G, Klein J L and Order S E 1987 Comparative biodistributions of yttrium- and indium-labeled monoclonal antibody B72.3 in athymic mice bearing human colon carcinoma xenografts Int. J. Radiation Oncology 14 1033-42

Msaki P, Axelsson B, Dahl C M and Larsson S A 1987 A generalized scatter correction technique in SPECT using point scatter distribution functions $J$. Nucl. Med. 28 1861-9

Msaki P, Axelsson B, Dahl C M and Larsson S A 1985 Improved scat ter correction technique in SPECT using point spread scatter functions Proc. 14th Int. Conf. Medical and Biological Engineering and 7th Int. Conf. on Medical Physics vol 23, suppl. 2, pp 1360-1

Singh $\mathrm{M}$ and Horne $\mathrm{C} 1988$ Use of a germanium detector to optimize scatter correction in SPECT $J$. Nucl. Med. 28 1853-60

Todd-Pokropek A, Clarke G, Marsch R, Gillardi M C and Fazio F 1984 sPECT quantification: The need for scatter and attenuation correction Radioaktive Isotope in Klinik und Forschung (Gasteiner Symp. 1984) vol 16, part 2, ed R Höfer and H Bergmann (Vienna: Egermann) 613-28

Yanch J C, Irvine A T, Webb S and Flower M A 1988 A comparison of deconvolution and window subtraction techniques for scatter compensation in SPECT IEEE Trans. Med. Imag. 7 13-20

Zasadny K R, Koral K F, Floyd Jr C E and Jaszcsak R J 1990 Measurement of Compton scattering in phantoms by germanium detector IEEE Trans. Nucl. Sci. NS-37 to appear 